\title{
Identification of Bacillus anthracis by a simple protective antigen-specific mAb dot-ELISA
}

\author{
K. S. R. Sastry, ${ }^{1}$ † U. Tuteja, ${ }^{1}$ P. K. Santhosh, ${ }^{2}$ M. K. Lalitha ${ }^{2}$ and H. V. Batra ${ }^{1}$ \\ ${ }^{1}$ Division of Microbiology, Defence Research and Development Establishment, Jhansi Road, Gwalior \\ 474 002, Madhya Pradesh, India \\ ${ }^{2}$ Department of Microbiology, Christian Medical College and Hospital, Vellore, Tamil Nadu, India
}

Correspondence

H. V. Batra

H_V_batra@rediffmail

Received 15 July 2002

Accepted 26 August 2002
A simple protective antigen (PA)-reactive mAb dot-ELISA was standardized for confirmation of toxinproducing strains of Bacillus anthracis. Twenty-seven clinical isolates were collected from patients clinically suspected of having anthrax. PA was elaborated from these isolates using Casamino acids medium and the culture medium was boiled to kill the cells. PA in boiled culture supernatants was detected using a dot-ELISA. Of the 27 clinical isolates tested, PA was detected in 24 isolates. This was further confirmed by amplifying the PA gene by PCR. This testing procedure is simple to perform, specific and safer than existing procedures, which are added advantages over existing methods of identification of $B$. anthracis. This test system could be a valuable tool in confirming clinical and environmental isolates of $B$. anthracis.

\section{INTRODUCTION}

Anthrax is widely distributed and cases have been reported from almost every country. Bacillus anthracis, the causative agent of anthrax, has two virulence-related plasmids, pXO1 and $\mathrm{pXO} 2$. pXO1 contains genes that encode the tripartite exotoxin, consisting of the components protective antigen $(\mathrm{PA})$, oedema factor (EF) and lethal factor (LF). These components individually are not toxic. The deleterious effects are due to the combined action of PA with either of the other two toxins. PA along with EF produces oedema, whereas the combination of PA and LF is lethal to susceptible animals and macrophage cell lines (Friedlander, 1986). The genes of $\mathrm{pXO} 2$ encode proteins involved in biosynthesis of the poly-D-glutamic acid capsule.

Preliminary identification of $B$. anthracis is usually made by microscopic examination of smears, colony morphology on nutrient or blood agar, the M'Fadyean reaction and motility test. Most isolates of $B$. anthracis from anthrax suspect cases can generally be identified by these characteristics. Sometimes, 'Bacillus cereus group' bacteria like B. cereus, Bacillus thuringiensis and Bacillus mycoides pose problems in identification as they are closely related to $B$. anthracis (Turnbull, 1999).

Confirmation of a virulent $B$. anthracis strain is usually carried out by inoculation of the suspect culture into mice or guinea pigs and/or by amplification of toxin and capsule

†Present address: Centre for Cellular and Molecular Biology, Uppal Road, Hyderabad 500 007, AP, India.

Abbreviations: EF, oedema factor; LF, lethal factor; PA, protective antigen. genes by PCR (Turnbull, 1998). These methods, though highly reliable, have some associated limitations. Here, we report a simple and specific PA-reactive mAb-based dotELISA for the confirmation of toxin-producing strains of $B$. anthracis.

\section{METHODS}

A total of 27 clinical isolates was collected from patients clinically suspected of having anthrax who were admitted to the Christian Medical College and Hospital, Vellore, India. Preliminary identification of these clinical isolates was carried out by microscopic examination of stained smears and colony morphology on nutrient and blood agar plates. All these isolates were subjected to PCR analysis using primers against a portion of the PA gene (Turnbull, 1998). For the demonstration of PA toxin, Casamino acids medium was prepared according to the reported protocol (Hains et al., 1965) except that charcoal was omitted from the medium. A loopful of each overnight-grown clinical isolate was separately inoculated into $50 \mathrm{ml}$ medium in a $250 \mathrm{ml}$ flask. Filter-sterilized glucose and sodium bicarbonate solutions were added to respective final concentrations of 0.2 and $0.9 \%$ at 0 and $4 \mathrm{~h}$. The flasks were incubated with constant shaking (100 r.p.m.) at $37^{\circ} \mathrm{C}$ for 24 h. Following incubation, the culture medium was boiled for $20 \mathrm{~min}$ to kill the cells and the cell debris was removed by centrifugation.

One $\mathrm{ml}$ of supernatant was collected from each flask and concentrated 20 -fold either by vacuum concentrator or by using pathopal particles (Ambica Enterprises). These concentrated culture supernatants were coated onto the nitrocellulose membrane tips of a plastic comb and blocked for $1 \mathrm{~h}$ at $37^{\circ} \mathrm{C}$ with $5 \%$ defatted milk in PBS. The combs were then washed with PBS and incubated with anti-PA mAbs (PA-2) for $1 \mathrm{~h}$ at $37^{\circ} \mathrm{C}$. Murine $\mathrm{mAbs}$ were generated against PA in the laboratory using the standard protocol with minor modifications (Kohler \& Milstein, 1975). After washing three times with PBST (PBS plus Tween $20,0.05 \%$ final concentration) for $20 \mathrm{~min}$, the combs were incubated with goat anti-mouse horseradish peroxidase-conjugate (Sigma) at 
1:1000 dilution for $1 \mathrm{~h}$. Following 15 min washing with PBST, the reaction was developed with $3,3^{\prime}$-diaminobenzidine dihydrochloride and hydrogen peroxide in PBS and then stopped by washing in tap water. The concentrated culture supernatants were also subjected to Western blotting as a further check for the presence of the $83 \mathrm{kDa} P A$ protein band (Towbin et al., 1979). The primary and secondary antibodies used for developing the nitrocellulose blot were the same as described in the dot-ELISA. In addition to the clinical isolates, the Sterne strain of B. anthracis and some other Bacillus species like B. cereus, Bacillus megaterium, Bacillus subtilis, B. mycoides and B. thuringiensis were also cultured and processed in the same manner.

\section{RESULTS AND DISCUSSION}

All 27 clinical isolates studied showed growth characteristics similar to those of B. anthracis. In dot-ELISA, of the 27 clinical isolates, a reaction was seen with concentrated culture supernatants of 24 isolates and also with the Sterne strain. None of the concentrated culture supernatants of other Bacillus species tested showed a positive reaction (Fig. 1). Similar results were obtained by Western blotting analysis (data not shown). Of the 27 clinical isolates, the same 24 that were positive for dot-ELISA were also positive for PA gene amplification ( $596 \mathrm{bp}$ ) by PCR, which further confirmed the presence of the PA gene in these isolates (Fig. 2).

Anthrax continues to be a problem in South India. Although there are occasional reports of human anthrax from other parts of India, there is considerable under-reporting, largely because of the lack of adequate microbiological facilities and test systems for the diagnosis of anthrax (Kumar et al., 2000). Being a potential agent of bioterrorism, there is a need and urgency to identify the organism unequivocally. Staining, the motility test and haemolysis on blood agar plates are routine methods used for presumptive identification of B. anthracis. Smears and cultural characteristics may not always be typical of $B$. anthracis. The motility test is one of the most useful procedures for preliminary screening of Bacillus isolates for $B$. anthracis. However, a recent report on the existence of motile $B$. anthracis strains from China has questioned its

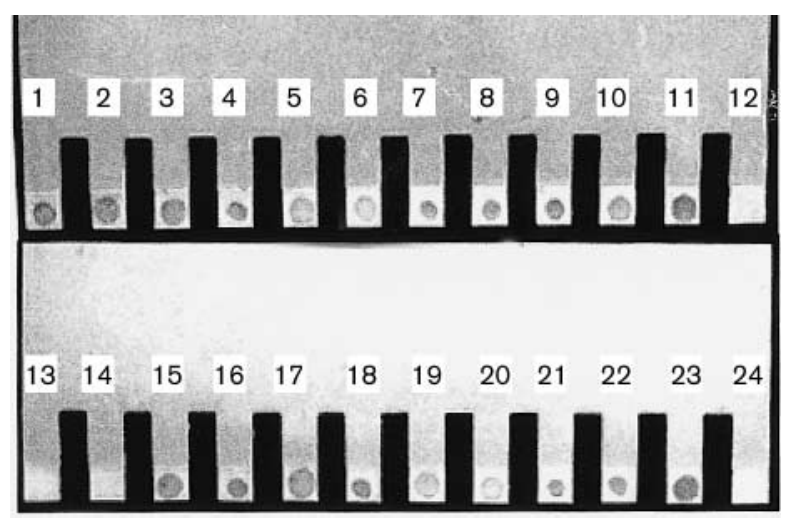

Fig. 1. Detection of $P A$ in concentrated culture supernatants by dotELISA. Samples: 1-10, clinical isolates $1-10 ; 11$ and 23, Sterne strain; 12, B. cereus; 13 and 14, clinical isolates 18 and 19; 15-22, clinical isolates $11-17$ and 20; 24, B. megaterium.

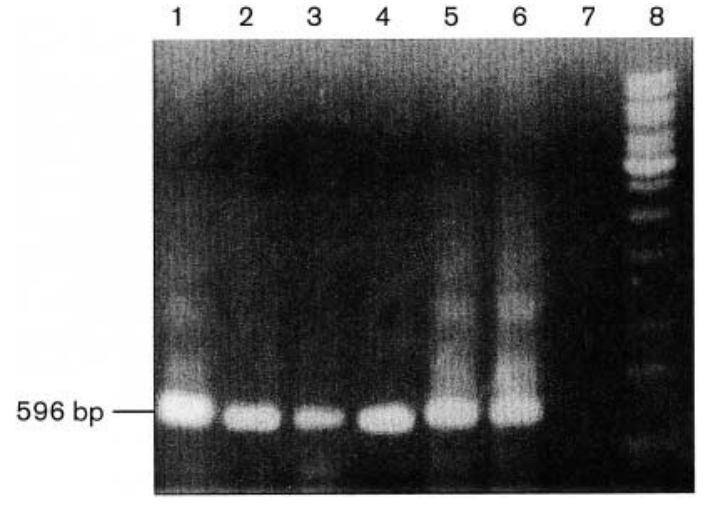

Fig. 2. Amplification of a 596 bp part of the PA gene by PCR. Lanes: 1, Sterne strain; 2-6, clinical isolates $1-5 ; 7$, B. cereus; 8,1 kb ladder.

validity in identification (Liang \& Yu, 1999). The gammaphage lysis assay, fluorescent-antibody staining and inoculation of the suspect culture into animals are conventionally utilized for confirmation of B. anthracis. In order to identify the organism, the phage must be used in conjunction with other tests, as phage-negative $B$. anthracis or phage-positive B. cereus are encountered occasionally (Turnbull, 1998). Fluorescent-antibody techniques are used to detect encapsulated organisms from culture. The limitation of this technique is cross-reaction with B. megaterium (Doyle et al., 1985).

Immunological and molecular-biological detection methods for $B$. anthracis offer the potential to be both more specific and more sensitive than cultural methods currently available. Over the years, numerous attempts have been made to identify anthrax-specific antigens and to design an antigenbased detection test for $B$. anthracis. However, most of the resulting systems are available only to highly specialized laboratories (Turnbull, 1999). PCR for PA, EF, LF and S-layer genes has been reported to be a confirmatory test (Turnbull, 1999). In some countries where the basic culture materials and reagents are not available and laboratories that are not equipped to conduct PCR, tests usually rely on animal inoculation, which is risky, particularly when proper biosafety protocols are not followed. These limitations are conveniently eliminated in the presently described $\mathrm{mAb}$ dotELISA for the confirmation of $B$. anthracis. The testing procedure is simple to perform, specific and relatively safer, which are added advantages over existing methods. Therefore, this dot-ELISA could be a valuable tool in confirming clinical as well as environmental isolates of $B$. anthracis containing the pX01 plasmid.

\section{ACKNOWLEDGEMENTS}

We thank Dr R. V. Swamy, Director, DRDE, Gwalior, for his keen interest in this work. 


\section{REFERENCES}

Doyle, R. J., Keller, K. F. \& Ezzell, J. W. (1985). Bacillus. In Manual of Clinical Microbiology, 4th edn, pp. 211-214. Edited by E. H. Lennette, A. Balows, W. J. Hausler, Jr \& H. J. Shadomy. Washington, DC: American Society for Microbiology.

Friedlander, A. M. (1986). Macrophages are sensitive to anthrax lethal toxin through an acid-dependent process. J Biol Chem 261, 7123-7126.

Hains, B. W., Klein, F. \& Lincoln, R. E. (1965). Quantitative assay for crude anthrax toxin. J Bacteriol 89, 74-83.

Kohler, G. \& Milstein, C. (1975). Continuous cultures of fused cells secreting antibody of predefined specificity. Nature 256, 495-497.
Kumar, A., Kanungo, R. \& Badrinath, S. (2000). Anthrax in Pondicherry, South India. J Med Microbiol 49, 1129.

Liang, X. \& Yu, D. (1999). Identification of Bacillus anthracis strains in China. J Appl Microbiol 87, 200-203.

Towbin, H., Staehelin, T. \& Gordon, J. (1979). Electrophoretic transfer of proteins from polyacrylamide gels to nitrocellulose sheets: procedures and some applications. Proc Natl Acad Sci U S A 76, 4350-4354.

Turnbull, P. C. B. (1998). Guidelines for the Surveillance and Control of Anthrax in Humans and Animals, 3rd edn. Geneva: World Health Organization.

Turnbull, P. C. B. (1999). Definitive identification of Bacillus anthracis a review. J Appl Microbiol 87, 237-240. 